\title{
In Situ Synthesis of Nanomaterials Using Environmental scanning/ Transmission Electron Microscope (ESTEM)
}

\author{
Renu Sharma \\ ${ }^{*}$ Center for Solid State Science, Arizona State University, Tempe, AZ 85287-1704
}

Nanomaterials are often synthesized using chemical vapor deposition (CVD) process. Therefore understanding the CVD process at nanoscale is required for selective synthesis of many nanostructures that can meet the challenges of the nanoworld. During CVD process a precursor molecule is decomposed to solid and gaseous components. The solid is deposited on a substrate while gaseous component is evacuated from the system. The decomposition energy can be either provided by heating the substrate or by light/ion/electron source. While the size of the nanopartcles can be controlled either way, only the photon, ion or electron sources can be used for site selective depositions. Scanning electron microscope (SEM) have been used form electron beam induced deposition (EBID) or for electron beam lithography for some time [1] but recently TEM is being used as it provides smaller probe size and better resolution to understand the reaction process at a nanometer level [2]. Similarly, carbon can be deposited by the decomposition of hydrocarbons on heated substrate (generally catalyst particles) to form carbon nanotubes (CNTs). These observations have provided valuable information about the CVD process. We have used an environmental scanning transmission electron microscope (ESTEM) to measure the kinetics of CVD processes under various reaction conditions.

Dynamic in situ observations were made using a specially modified environmental scanning/transmission electron microscope (ESTEM), a Tecnai F20, operated at 200KV, equipped with a Gatan imaging filter (GIF) and annular dark-field detector [3]. Organometallic precursors (for example $\left(\mathrm{CH}_{3}\right)_{2}\left(\mathrm{C}_{5} \mathrm{H}_{7} \mathrm{O}_{2}\right) \mathrm{Au}$ for gold) were used as source for deposition of metal particles on $\mathrm{SiO}_{2}$ thin films whereas acetylene (hydrocarbon) source was used to deposit carbon on $\mathrm{Ni} / \mathrm{SiO}_{2}$ catalyst for CNT synthesis. Metal particles were selectively deposited by EBID using various probe size at different temperatures. Time resolved images were recorded using 1024x1024 Gatan CCD and/or digital video camera. STEM images were recorded to determine the particle size distribution. On the other hand, the catalyst samples were heated up to $400-700^{\circ} \mathrm{C}$ using a furnace heating holder in vacuum $(\approx 10-6$ Torr $)$ or 2 Torr of $\mathrm{H}_{2} .10-100 \mathrm{mTorr}$ of acetylene $\left(\mathrm{C}_{2} \mathrm{H}_{2}\right)$ was slowly leaked into the sample region at the reaction temperature, and bright-field images were recorded using a digital video recorder connected to a TV rate camera.

The diameter of the deposited metal particles depended upon the electron probe size but was always larger than the diameter of the electron probe due to secondary electron effects [4]. For example, the probe size of $7 \mathrm{~nm}$ diameter produced a gold dot of 35 (Figure 1a) and the difference in the diameter of the probe and Au dot was nearly identical for different probe sizes [5]. Time resolved images (Figure 1b-c) were used to obtain the rate of the Au deposited (Figure 1d) and was found to taper off with time as the number of secondary electron, responsible for providing energy for decomposition, escaping to the surface decreased with increased thickness of the deposited materials. The deposition rate decreased with increased temperature as the sticking coefficient of precursor decreased at higher temperature.

Similarly, the CVD process for the formation of CNT can be followed using the same ESTEM.

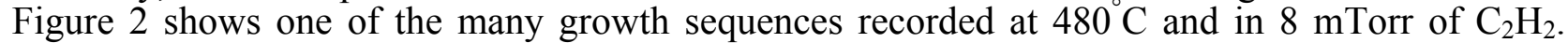
Under these conditions most of the tubes were observed to be anchored at both ends to the substrate. One end detached after growing for a few seconds [6]. Sometimes, tubes were observed to grow straight out of the substrate (Figure 2a-c). The tip of the tube had a hollow round structure (figure $3 \mathrm{~b}-3 \mathrm{c}$ ) indicating the absence of a Ni particle at the tip. Temperature and pressure resolved growth rates of carbon nanotubes were obtained from the individual frames digitized from the video sequence. The growth rates measured in this manner were broadly distributed, and 
indicative of discontinuous growth. SWCNTs were observed to form under these synthesis conditions (Figure 3). Our data show that the growth rates, duration of growth, and length of the CNTs formed, are controlled by the synthesis conditions.

Thus we have been able to synthesize metal particles and CNTs using the ESTEM to obtain a better understanding of the electron beam induced and thermally induced CVD processes. In future we will combine the two processes to deposit catalyst particle using EBID and observe the formation of CNTs from these particles in situ to obtain a better understanding of the catalyst surface and CNT nucleation and growth mechanism that will be discussed in detail.
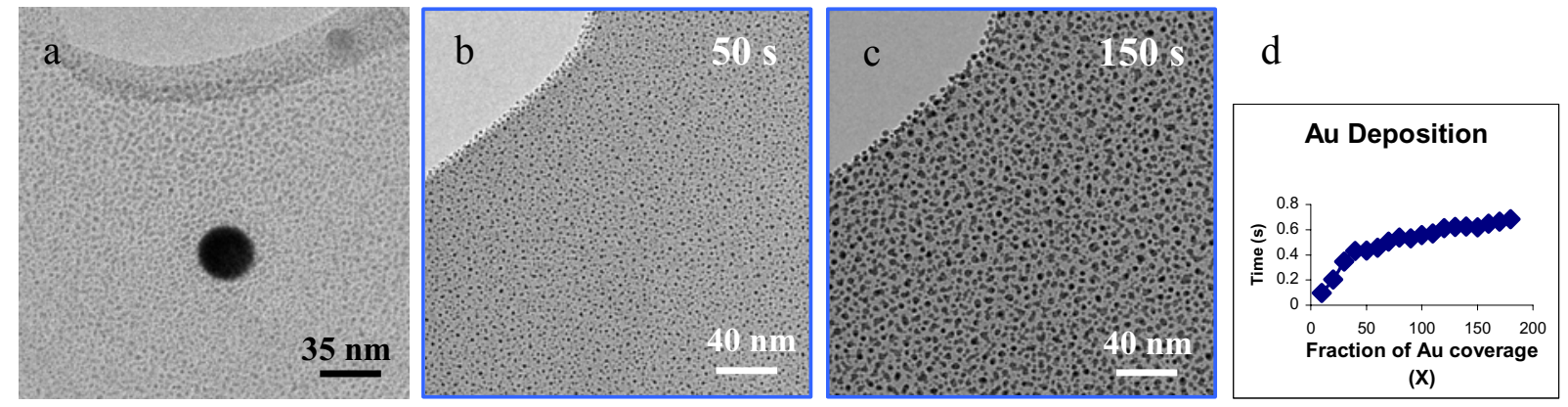

Figure 2. (a) $35 \mathrm{~nm}$ Au dot was deposited using $7 \mathrm{~nm}$ probe diameter. (b-c) Time resolved TEM images were used to (d) estimate the rate Au of deposited. The deposition time is marked in upper right hand corner of the images.

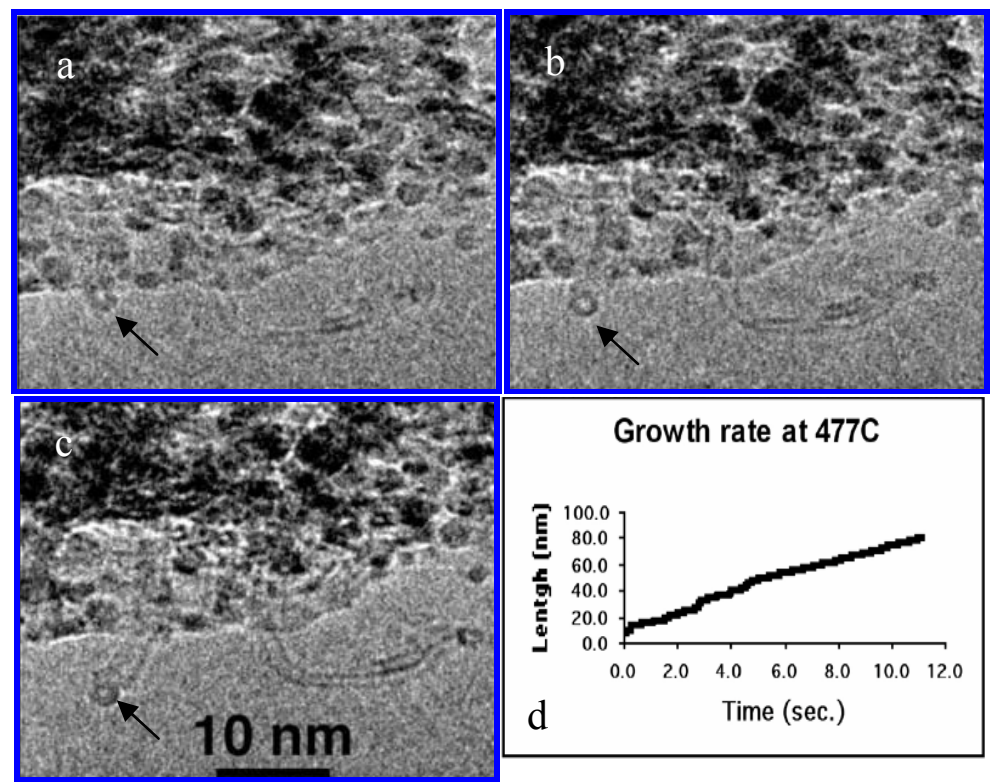

\section{References:}

[1] E.E.Ehrichs, et al. Appl.Phys.Lett. 53, (1988) 2287.

[2] Williem F. van Dorp et al. Nano Lett.5 (2005) 1303.

[3] Renu Sharma, J. Mat. Res. 20 (2005) 1695.

[4] N. Silvis- Cividjian et al. Appl.Phys.Lett. 82, (2003) 3514.

[5] S. Ketharanathan et al. J. Vac. Sci. Tech B23 (2005) 2403

[6] Renu Sharma et al, J. Eelectron Microsc. 54 (2005) 231.

[7] Financial support from NSF-CTS\# 0508434 and use of the John Cowley Center for High Resolution Electron Microscopy is gratefully acknowledged.

Figure 3. Time resolved images obtained from a digital video sequence showing the growth of a straight CNT. The time lapsed between $a$ to $b$ and $c$ is 1.5 and 3.5 seconds respectively. (d) This digital sequence was used to obtain instantaneous growth rates. Note that there are other nanotubes that were observed to form a few seconds earlier.

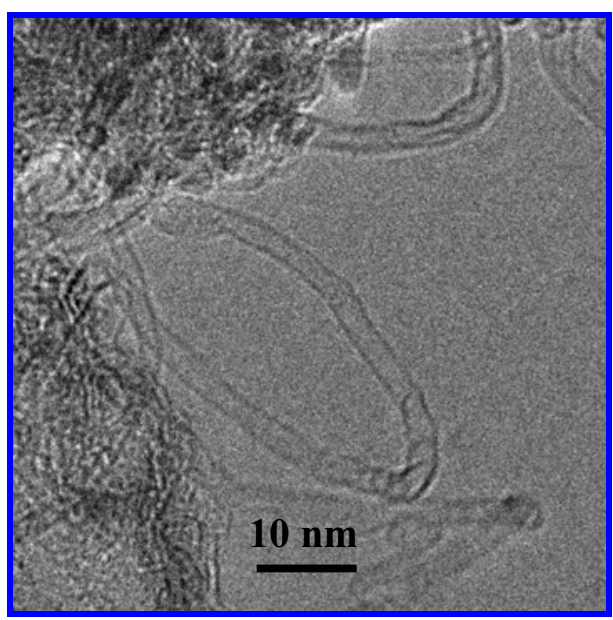

Figure 3. High resolution image of SWCNT at 520C in 8mTorr of $\mathrm{C}_{2} \mathrm{H}_{2}$. 